\title{
Usefulness of MALDI-TOF mass spectrometry in epidemiological control of etiologic agents of infection
}

\author{
Roberto Degl'Innocenti,Tamara Brunelli, Lorenzo Pellegrini, Daniele Ferri, Elisa Rovinati, \\ Michele Rispoli, Antonella Conti, Patrizia Casprini \\ Laboratorio Analisi Chimico Cliniche, Ospedale Misericordia e Dolce, ASL4, Prato
}

Key words: mass-spectrometry, epidemiology, Staphylococcus epidermidis, Staphylococcus aureus

Uso della spettrometria di massa MALDI-TOF nel controllo epidemiologico di agenti eziologici di infezione

\section{SUMMARY}

Introduction: The use of the MALDI-TOF mass spectrometry in the routine of microbiological diagnostics has revolutionized procedures and response times of bacteriology. The use of this technique aims to epidemiological investigations in a hospital environment and represents a further significant opportunity, quickly feasible and extremely economical.

Methods: By means of the MALDI-TOF-MS Vitek2 (MS Vitek2) mass spectrometer, accompanied by the AgnosTec-SARAMIS (bioMérieux, France) software, were analyzed the spectra of 149 bacterial isolates (I39 Staphylococcus aureus and 10 Staphylococcus epidermidis) obtained from cultures of I48 patients (I4I inpatients and 7 outpatients). Clinical isolates were stored at a temperature of $-20^{\circ} \mathrm{C}$. The isolates were then thawed and immediately cultured on agar blood medium. The colonies were subjected to analysis by MS Vitek on the day after sowing. The spectra obtained were analyzed and compared using the software AgnosTec-SARAMIS, which allowed the construction of a dendrogram.

Results and conclusions: The evaluation of the data collected suggests that mass spectrometry could be an useful tool in epidemiological surveys. Speed of analysis and low costs make the MS Vitek2 an usable tool by many microbiology laboratories.

\section{INTRODUZIONE}

L'introduzione della spettrometria di massa MALDI-TOF nella routine diagnostica microbiologica ha rivoluzionato le procedure ed $\mathrm{i}$ tempi di risposta relativi agli esami batteriologici (1-3). L'utilizzo della spettrometria di massa, finalizzato alle indagini epidemiologiche in ambito ospedaliero, potrebbe rappresentare un'opportunità da non sottovalutare, sulla base dei numerosi vantaggi sia in termini di rapidità che da un punto di vista economico (5).

Al fine di valutare le potenzialità dell'applicazione di questa tecnologia in campo epidemiologico, abbiamo analizzato 139 ceppi di Staphylococcus aureus (S.aureus) e 10 ceppi di Staphylococcus epidermidis (S.epidermidis) raccolti dall'anno 2008 ad oggi. I campioni biologici (prevalentemente sangue), conservati presso il laboratorio, provenivano sia da pazienti ospedalizzati che ambulatoriali afferenti al nostro ospedale.

Abbiamo voluto inoltre indagare, nel caso di una sospetta sepsi catetere-correlata (CatheterRelated Bloodstream Infection CRBSI), l'affida- bilità di questo strumento nell'indagare le relazioni clonali fra i ceppi di stafilococco coagulasi negativi $(\mathrm{SCN})$ isolati da catetere venoso centrale (CVC) e quelli da provenienti da vena periferica dello stesso paziente.

\section{MATERIALI E METODI}

Sono stati analizzati gli spettri di 139 ceppi di $S$. aureus, di cui 132 isolati da emocoltura di degenti dei reparti di Geriatria, Malattie Infettive, Nefrologia, Oncologia, Rianimazione, Chirurgia, Ortopedia e di una Clinica Privata convenzionata, e 7 da campioni vari (ulcere, urine, materiale respiratorio) di pazienti ambulatoriali.

Sono stati inoltre analizzati 10 ceppi di S. epidermidis, di cui 2/10 isolati da sangue (prelievo venoso e CVC) di uno stesso paziente ed 8/10 da campioni ematici di 8 diversi pazienti e reparti.

Gli spettri dei ceppi batterici sono stati analizzati il giorno stesso dell'isolamento colturale oppure successivamente, previo scongelamento dello stock a $\mathrm{T}=-20^{\circ} \mathrm{C}$.

Se analizzati in un secondo tempo gli isolati sono

\section{Corresponding author: Tamara Brunelli}

U.O. Analisi Chimico Cliniche, Ospedale Misericordia e Dolce, USL4, Prato

Piazza dell'Ospedale 5, 59100 Prato - Tel:+39-0574-4347/9

E-mail: tbrunelli@usl4.toscana.it 
stati seminati su terreno al sangue (bioMérieux, France) ed incubati a $37^{\circ} \mathrm{C}$ per 18 ore. Il pozzetto per eseguire l'analisi su Vitek MS (Axima Shimadzu - bioMérieux, France) è stato allestito il giorno successivo alla semina in coltura.

Gli spettri ottenuti sono stati analizzati e confrontati con l'utilizzo del software AgnosTec-SARAMIS (bioMérieux, France) per la costruzione di un dendrogramma (4).

\section{RISULTATI}

Tutti i ceppi batterici sono stati identificati correttamente mediante il sistema Vitek MS, con una confidenza superiore o uguale al $90.00 \%$.

Gli spettri sono stati quindi importati e analizzati mediante il software SARAMIS.

Reparto di Malattie Infettive (Figura I):

gli isolati sono risultati essere ascrivibili principalmente a quattro cluster (.......), che farebbero parte di un gruppo più ampio $(\square)$ e comprendente ceppi aventi una similarità pari ad oltre il $75 \%$.

L'analisi epidemiologica ha reso quindi possibile ipotizzare la presenza, all'interno del reparto, di un unico clone endemico.

Reparto di Geriatria (Figura II):

in questo reparto sono stati individuati 4 cluster (......), 2 dei quali (...) ascrivibili ad un gruppo più ampio $(\square)$ predominante numericamente sugli altri: è risultato infatti rappresentare circa il 50\% degli isolati.

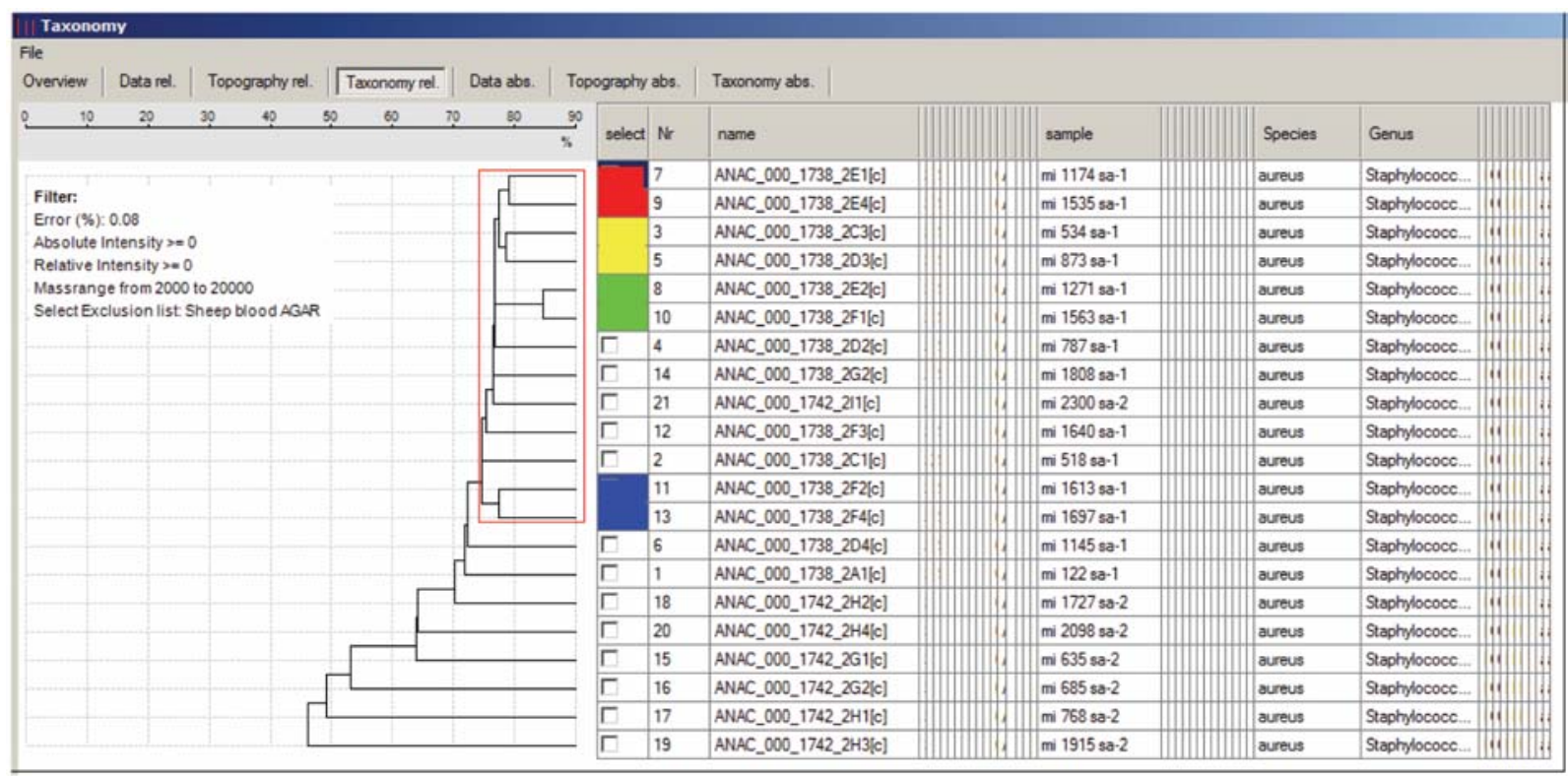

Figura I. Isolati di S.aureus nel reparto di Malattie Infettive.

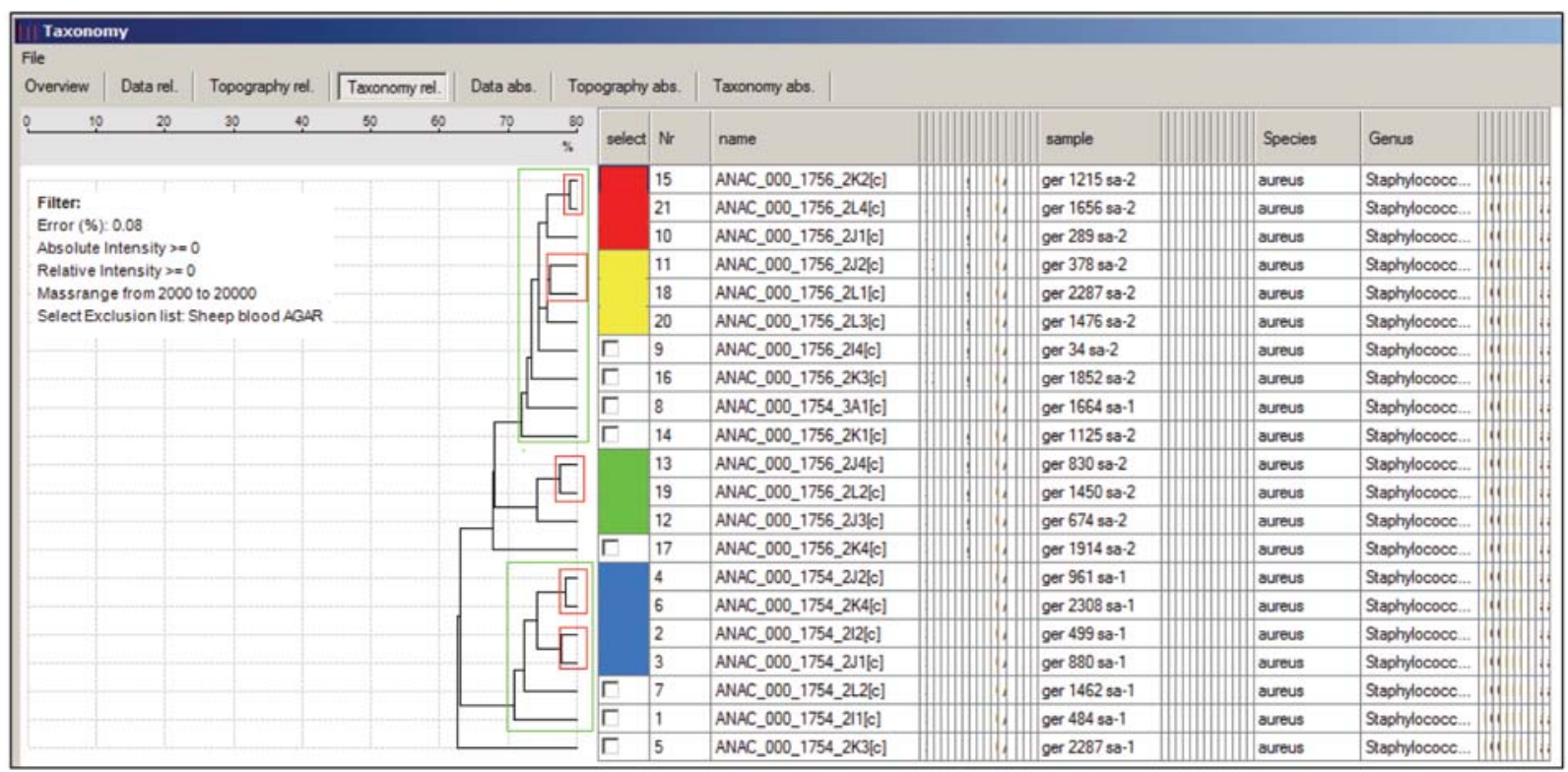

Figura II. Isolati di S.aureus nel reparto di Geriatria. 
Reparto di Nefrologia (Figura III): è risultato predominarvi un clone fortemente rappresentato ( $\mathrm{\square})$, comprendente circa il 70\% degli isolati. È stato possibile evidenziare una somiglianza superiore al $70 \%$ fra i ceppi del gruppo, all'interno del quale è stato possibile evidenziare due sottogruppi minori composti da 3 (..) e 2 (..) isolati.

Reparto di Rianimazione (Figura IV): in questo reparto è sembrata prevalere una linea clonale consistente $(\square)$ comprendente isolati simili fra loro per oltre il $70 \%$. È importante sottolineare come i ceppi di $S$. aureus provenienti da pazienti non ospedalizzati (Pazienti 1, 2, 3, 4, 5, 6 e 7) siano risultati differire in modo sostanziale da quelli raccolti presso il reparto di Rianimazione (visibili nella parte superiore del dendrogramma).

Reparto di Oncologia (Figura V): anche in questo reparto è stato possibile osservare un ampio gruppo di isolati $(\square)$ con similarità di spettro superiore al $75 \%$. All'interno del clone sono risultati evidenziabili tre sottogruppi omogenei (similarità superiore all' $80 \%$ ) (......).

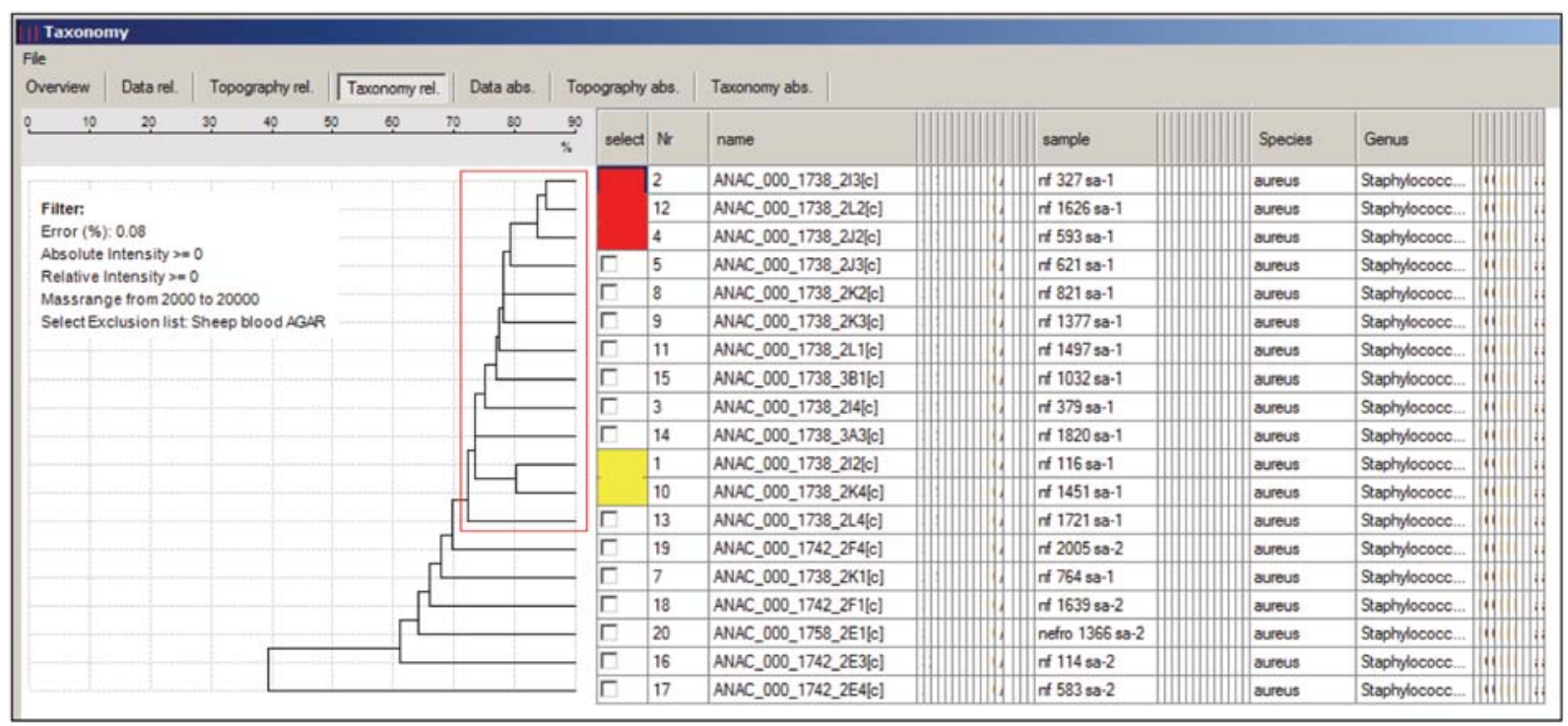

Figura III. Isolati di S.aureus nel reparto di Nefrologia.

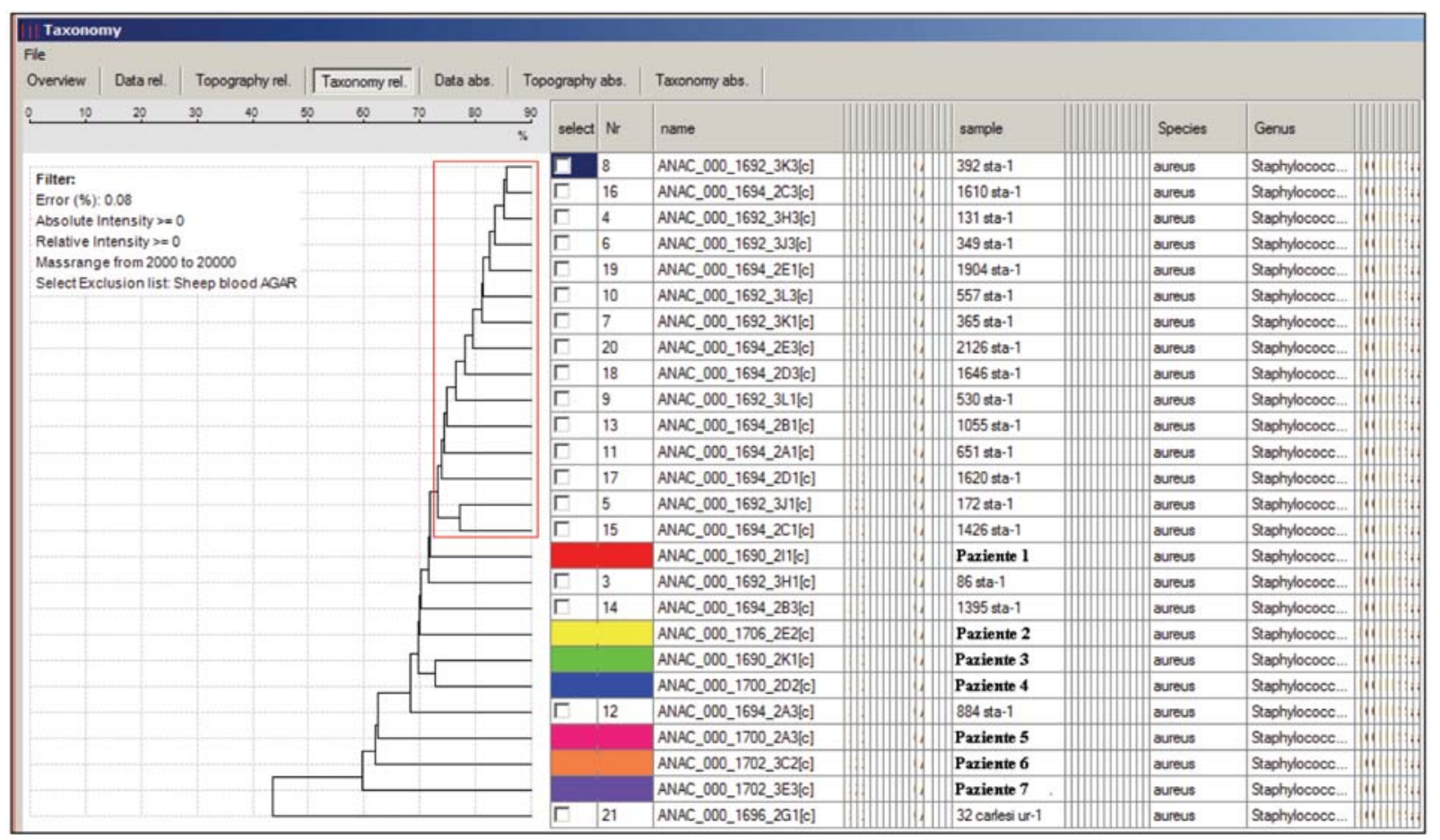

Figura IV. Isolati di S.aureus nel reparto di Rianimazione. 


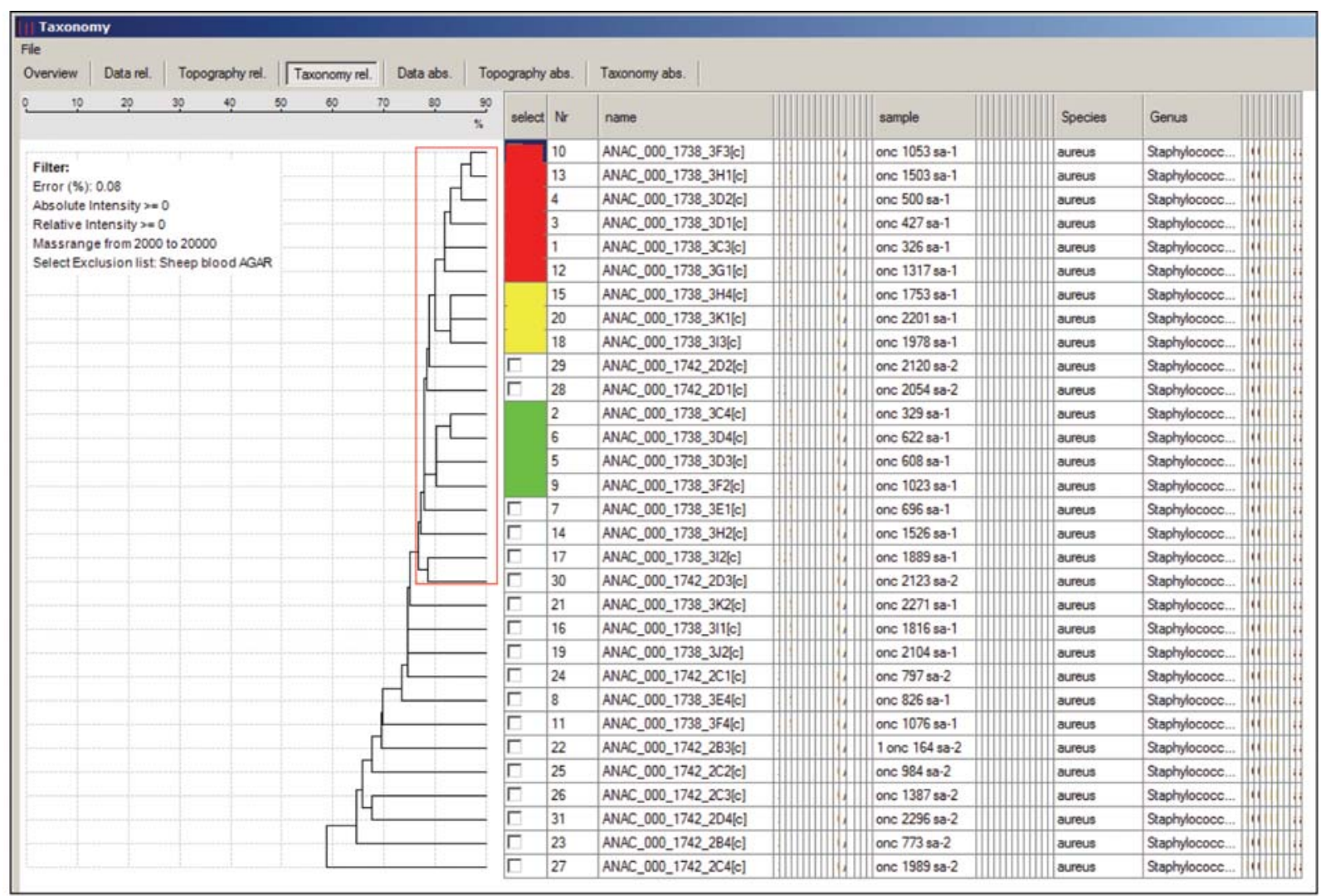

Figura V. Isolati di S. aureus nel reparto di Oncologia.

Reparto di Ortopedia (Figura VI): tutti gli isolati sembravano appartenere (ad eccezione del ceppo 2371) ad un unico clone (..), con una similarità di spettro superiore al $70 \%$.

Reparto di Chirurgia (..) e Clinica Privata (..) (Figura VII): in questo caso è stato possibile osservare come lo spettro del gruppo degli isolati provenienti dal reparto di Chirurgia (..) fosse completamente diverso da quello relativo agli isolati della Clinica Privata (..), e come questi ultimi non fossero affatto omogenei fra loro. Ciò avvalore- rebbe l'ipotesi della presenza di un cluster di $S$. aureus persistente all'interno del reparto di Chirurgia.

In Figura VIII è possibile osservare come i due ceppi di $S$. epidermidis isolati da vena periferica e CVC del paziente 1 (PZ1 .) (in un caso di sospetta CRBSI) siano risultati simili per 1' $80 \%$.

Questi ultimi ceppi, sono risultati significativamente differenti da quelli provenienti dai campioni ematici di altri degenti (somiglianza inferiore al $65 \%$ ).

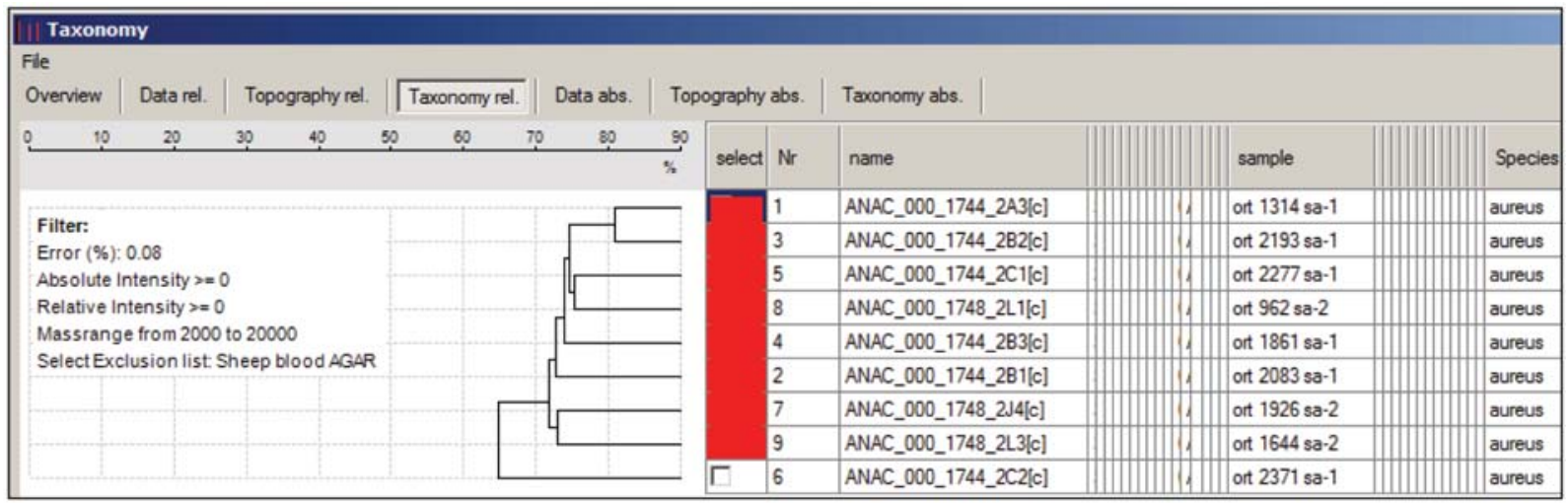

Figura VI. Isolati di S.aureus nel reparto di Ortopedia. 


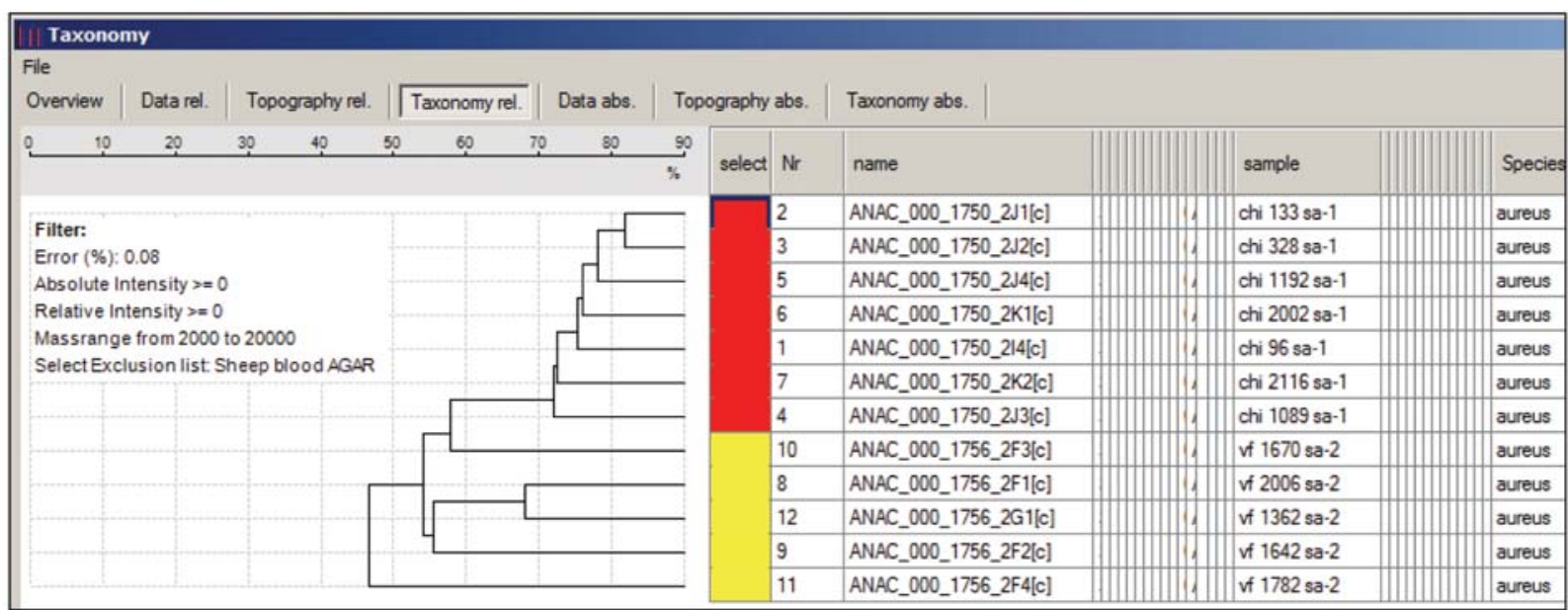

Figura VII. Isolati di S. aureus dalla Chirurgia e da una Clinica Privata.

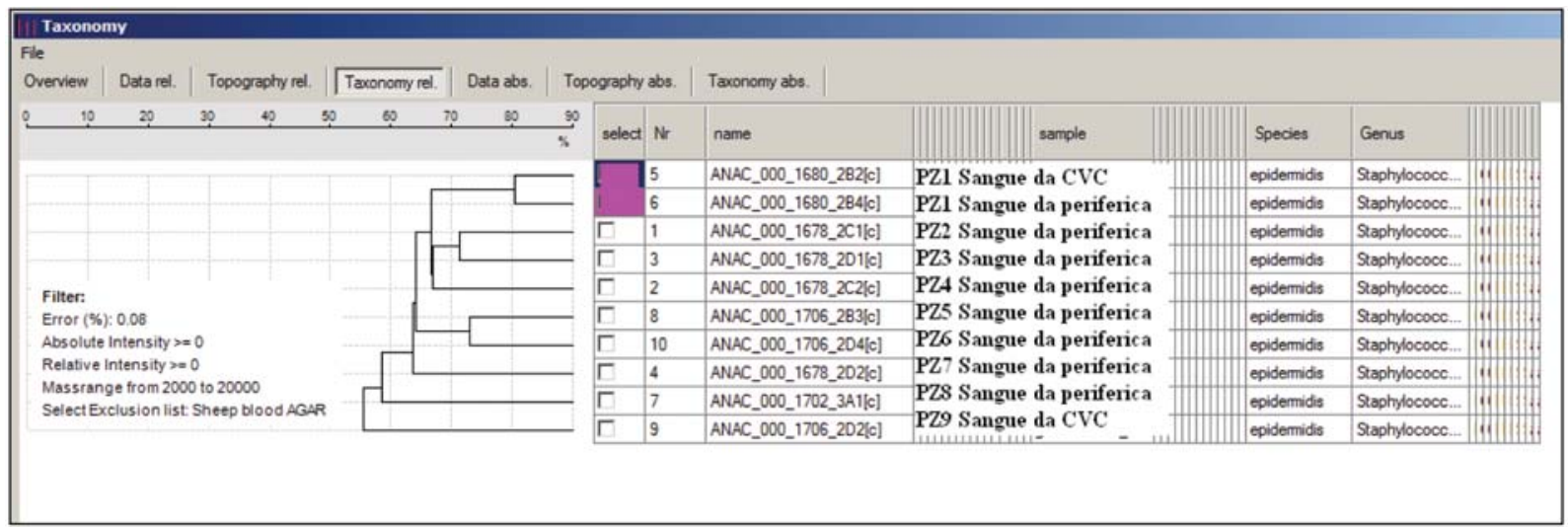

Figura VIII. Isolati di S. epidermidis da campioni ematici (vena periferica o/e CVC).

\section{DISCUSSIONE}

Poter individuare, mediante uno strumento sensibile, economico e di facile utilizzo, cluster epidemici può essere un valido aiuto nello studio e nel controllo della diffusione degli agenti eziologici di infezione in ambiente nosocomiale. Il sistema Vitek MS se utilizzato quotidianamente, consentirebbe di riconoscere in tempo reale la diffusione di particolari cloni batterici nelle aree a rischio, permettendo un rapido intervento di contenimento (5). In tutti i reparti da noi considerati, è stato possibile osservare la presenza di cluster più o meno importanti numericamente; ciò ha permesso di ipotizzare il persistere nel tempo, nelle aree considerate, di alcune specie batteriche.

Una considerazione particolare deve essere fatta per l'analisi degli isolati di $S$. epidermidis in un caso di sospetta CRBSI. È infatti noto come, anche se il tempo di positivizzazione fra un flacone prelevato da CVC rispetto a quello prelevato da vena periferica è inferiore alle 2 ore, gli SCN isolati non debbano appartenere necessariamente alla stessa specie e/o allo stesso biotipo (potendo due biotipi diversi della stessa specie essere responsabili della contaminazione dei due prelievi). Fino ad oggi, dirimere questo dubbio risultava possibile solo con l'utilizzo di metodi molecolari e quindi con alti costi, tempi di attesa lunghi e con il coinvolgimento di personale altamente specializzato (6). L'elevata similarità di spettro $(80 \%)$ riscontrata fra i due isolati ottenuti rispettivamente da CVC e da vena periferica del Paziente 1 e la completa diversità rispetto agli altri ceppi (somiglianza inferiore al 65\%), hanno reso superflua ogni altra tecnica di conferma e permesso di accertare la relazione di clonalità fra i primi due isolati.

\section{CONCLUSIONI}

I dati riportati suggeriscono che il sistema utilizzato nella presente esperienza può rappresentare uno strumento di utile impiego sia in indagini di tipo epidemiologico che in tutti i casi in cui sia necessario verificare la similarità fra due o più isolati batterici (come ad esempio nelle CRBSI). Rapidità di analisi e costi contenuti rendono la spettromeria di massa senza dubbio uno strumento versatile ed alla portata di molti Laboratori di Microbiologia Clinica. 


\section{BIBLIOGRAFIA}

1. Bizzini A, Greub G. Matrix-assisted laser desorption ionization time of flight mass spectrometry, a revolution in clinical microbial identification. Clin Microbiol Infect 2010; 16: 1616-7.

2. Drancourt M. Detection of microorganisms in blood specimens using matrix-assisted laser desorption time-of flight mass spectrometry: a review. Clin Microbiol Infect 2010; 16: 1620-5.

3. Lartigue MF. Matrix-assisted laser desorption ionization time-of-flight mass spectrometry for bacterial strain characterisation. Inf Gen Evol 2013; 13: 230-5.
4. Martiny D, Busson L, Wybo I, El Haj RA, Dediste A, Vandenberg O.Comparison of the Microflex LT and Vitek MS systems for routine identification of bacteria by matrix-assisted laser desorption ionization-time of flight mass spectrometry. J Clin Microbiol 2012; 50: 1313-25.

5. Murray PR. Matrix-assisted laser desorption ionization time-of-flight mass spectrometry: usefulness for taxonomy and epidemiology. Clin Microbiol Infect 2010; 16: 1626-30.

6. Murray PR, Masur H. Current approaches to the diagnosis of bacterial and fungal bloodstream infections in the intensive care unit. Crit Care Med 2012; 40: 3277-82. 